\title{
UNA NUEVA ESPECIE DE PELLAEA (PTERIDACEAE) DEL ESTADO DE SAN LUIS POTOSÍ, MÉXICO
}

\author{
Aniceto Mendoza \\ Departamento de Biología, C.B.S. \\ Universidad Autónoma Metropolitana, Unidad Iztapalapa \\ Apartado postal 55-535 \\ 09340 México, D.F. \\ e-mail: amr@xanum.uam.mx \\ MIKE WINDHAM \\ Utah Museum of Natural History \\ University of Utah \\ 1390 E Presidents Circle \\ Salt Lake City, Utah 84112 U.S.A. \\ Blanca Pérez-García \\ Departamento de Biología, C.B.S. \\ Universidad Autónoma Metropolitana, Unidad Iztapalapa \\ Apartado postal 55-535 \\ 09340 México, D.F. \\ Y \\ George YATSKIEVYCH \\ Missouri Botanical Garden \\ P.O. Box 299 \\ St. Louis, MO 63166-0299 U.S.A.
}

\section{RESUMEN}

Se describe e ilustra Pellaea ribae Mendoza \& Windham, una nueva especie de Pellaea sect. Pellaea la cual se distingue claramente de otros representantes del género por sus abundantes tricomas blanquecinos presentes en el pecíolo, en el raquis y en la superficie abaxial de la lámina. Hasta ahora $P$. ribae se conoce solamente de dos localidades en San Luis Potosí, México. Se discuten las posibles relaciones de esta nueva especie con otros helechos cheilantoides.

Palabras clave: Pellaea, Pteridaceae, México. 


\section{ABSTRACT}

A new species of Pellaea sect. Pellaea is described and illustrated, Pellaea ribae Mendoza \& Windham, which is clearly distinguished from other species in the genus by the abundant white trichomes present on the petiole, rachis, and abaxial surface of the leaves. Presently $P$. ribae is known only from two localities in San Luis Potosí, Mexico. Possible relationships of this new species to other cheilanthoid ferns are discussed.

Key words: Pellaea, Pteridaceae, Mexico.

Pellaea Link sensu lato agrupa alrededor de 35-40 especies que se distribuyen ampliamente en las zonas áridas y semiáridas del país. El género tiene sus centros de diversidad en el suroeste de los Estados Unidos, México, América del Sur y sur de África (Anthony, 1984; Tryon y Tryon, 1982; Windham, 1993a). La sección Pellaea (Pellaea sensu stricto) comprende alrededor de 20 especies y está casi restringida al continente americano, con una gran diversidad en México y el suroeste de los Estados Unidos. Varios taxa han sido descritos recientemente (Windham, 1993) y otras novedades esperan su reconocimiento.

En un viaje de trabajo al estado de San Luis Potosí, el primer autor recolectó ejemplares de una población de Pellaea, el cual nos llamó la atención por sus abundantes tricomas de color blanquecino en prácticamente toda la hoja. Después de una revisión minuciosa del material y de un análisis cuidadoso de la literatura, llegamos a la conclusión de que se trata de un taxon no descrito, por lo que proponemos:

Pellaea ribae Mendoza \& Windham, sp. nov. Figs. 1, 2.

Pellaeae atropurpureae (L.) Link et $P$. ternifoliae (Cav.) Link subsp. villosae Windham similis, a quibus differt stipitibus rhachidibusque dense villosis abaxialiter et adaxialiter, pinnis indivisis ubique et dense villosis abaxialiter; insuper a $P$. ternifolia subsp. villosa differt paleis rhizomatum concoloribus, pinnis sine mucrone, sporis plus quam $53 \mu \mathrm{m}$ longis metientibus.

Hierba perenne, epipétrica, raramente terrestre. Rizoma cortamente rastrero, de 6$10 \mathrm{~mm}$ de diámetro; escamas del rizoma de $0.8-1.5 \mathrm{~cm}$ de largo, filiformes con los márgenes escasamente denticulados, flexuosamente atenuadas en el ápice, concoloras, de color pardo-amarillento a leonado. Hojas pinnadas, de $10-40 \mathrm{~cm}$ de largo, monomorfas; prefoliación densamente vilosa. Pecíolo y raquis rollizos, no flexuosos, pardos a púrpuranegruzcos, sin articulaciones prominentes en las líneas proximales, densamente pubescentes con tricomas hialinos a blanquecinos; los tricomas pluricelulares, uniseriados, de 3-9 $\mathrm{mm}$ de largo. Lámina linear-lanceolada, 1-pinnada. Pinnas de 1-2.8 cm de largo, linearoblongas, sésiles, por lo general algo deflexas, opuestas a subopuestas, ocasionalmente alternas a lo largo de todo el raquis, con las bases cordadas y los ápices redondeados (no mucronados), adaxialmente glabros, densamente viloso-tomentosos en la superficie abaxial; márgenes de las pinnas enteros, ligera a fuertemente recurvados en la pinna fértil, con un borde blanquecino muy bien definido. Venación libre, venas 1-2 veces divididas dicotómicamente. Soros continuos, a todo lo largo de los márgenes de las pinnas. 


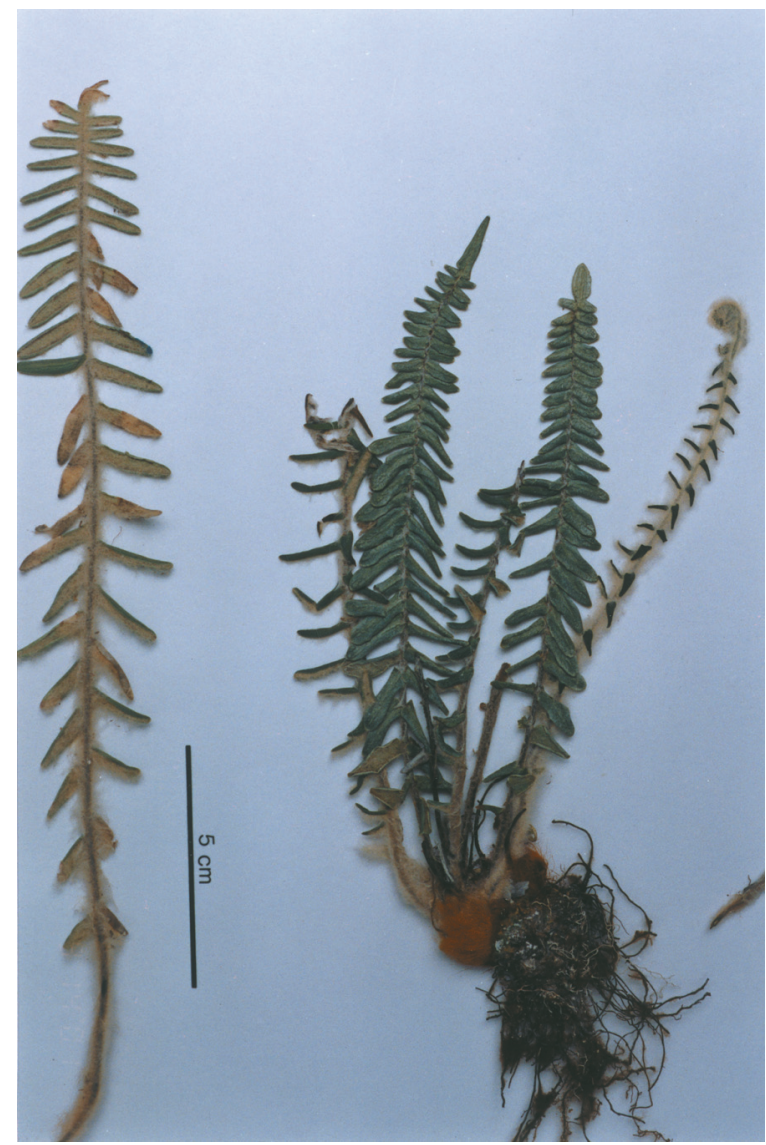

Fig. 1. Pellaea ribae Mendoza \& Windham. Hábito de la planta y vista abaxial de la pinna; obsérvese la presencia de abundantes tricomas en toda la planta.

Esporangios esparcidos o poco diferenciados en las puntas de las venas, muy cortamente pedicelados (subsésiles), conteniendo 64 esporas, parafisos ausentes. Esporas de 53 (55) $60 \mu \mathrm{m}$ de diámetro, triletes, tetraédrico-globosas a globosas, perina rugulada a rugosa, crestada, a menudo equinulada, de color pardo oscuro.

Hábitat: Laderas y paredes rocosas sobre suelos yesosos y derivados de yeso, en comunidades de matorral xerófilo; altitud 1260-1350 m.

Tipo: San Luis Potosí, municipio de San Nicolás Tolentino, $3 \mathrm{~km}$ después del Puerto de Santa Gertrudis, rumbo a Buenavista, 22011'27" N, 100²0'56" W, 1260 m s.n.m., matorral 


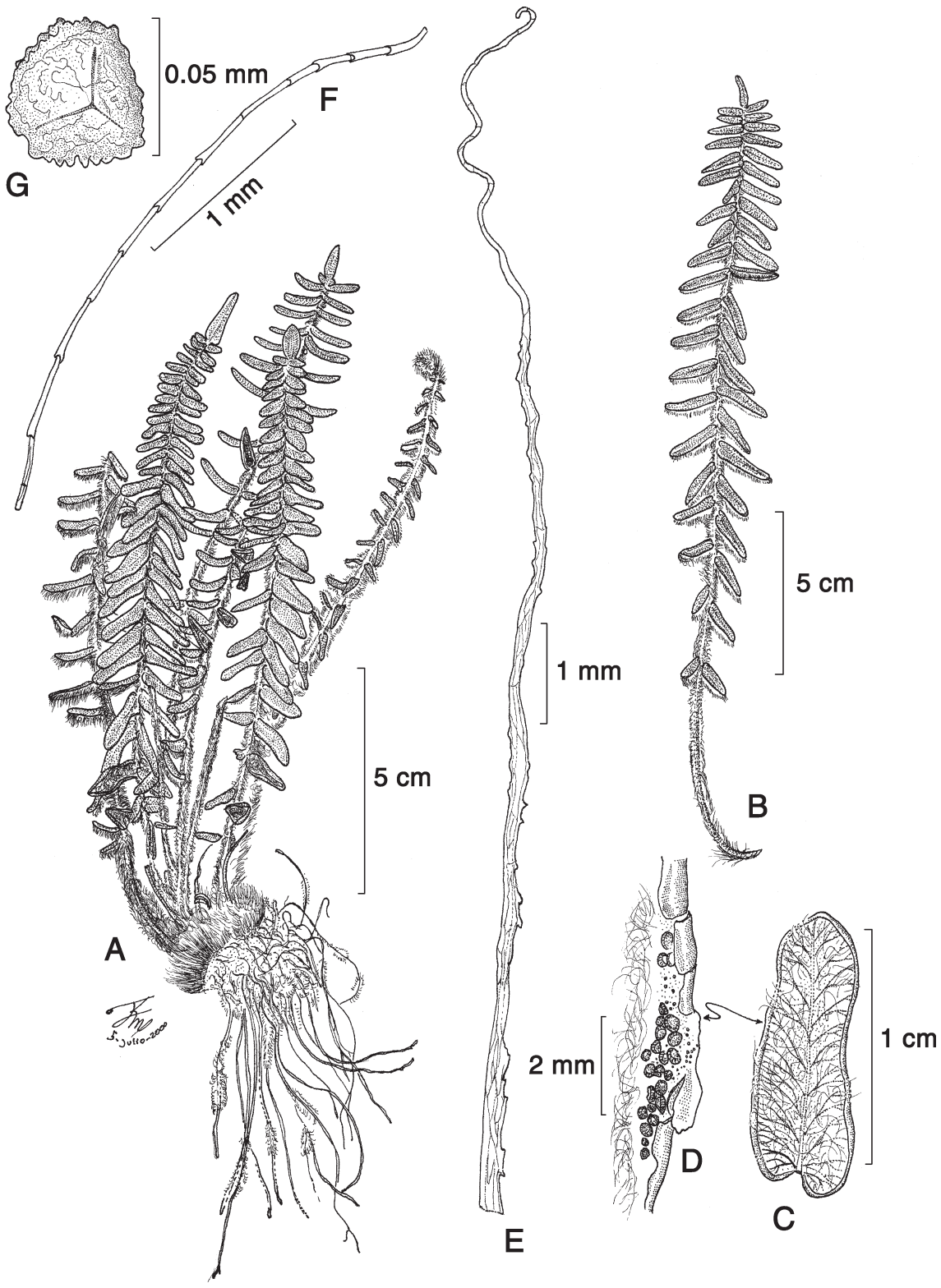

Fig. 2. Pellaea ribae Mendoza \& Windham. A. Planta completa; B. Hoja completa de la misma planta; C. Acercamiento de la cara abaxial de una pinna, con su venación y tricomas; D. Detalle del soro marginal; E. Escama del rizoma; F. Tricoma pluricelular uniseriado; G. Espora trilete. 
xerófito, 15.XI.1997, A. Mendoza R. (250), J. Ceja, A. Espejo y A. R. López Ferrari (holotipo: UAMIZ, isotipos IEB, MO, NY, SLPM y UT, por distribuir).

Paratipo: San Luis Potosí, ca. 11 mi S of Villa Juarez at pass; side canyon of gypsum, 25.V.1989, K. Heil 5347 (BRY).

El nombre de este taxon está dedicado en honor del Dr. Ramón Riba y Nava Esparza (1934-1999), quien fuera el más destacado pteridólogo mexicano.

La nueva especie se distingue de todos los demás representantes del género Pellaea que crecen en México, por la presencia de abundantes tricomas pluricelulares uniseriados, de color blanquecino, distribuidos en el pecíolo, en el raquis y en la cara abaxial de las pinnas, y por presentar la lámina una vez pinnada (Figs. 1-2). Las dos poblaciones conocidas están ubicadas aproximadamente a $12 \mathrm{~km}$ una de otra; así Pellaea ribae es un taxon endémico raro y de área reducida, en la cual la existencia de minas de yeso son una continua amenaza para su supervivencia.

Pellaea ribae es un taxon evidentemente distinto, cuya relación con las otras especies de Pellaea no es inmediatamente evidente. De hecho, el espécimen paratipo en BRY no había sido identificado ni siquiera a nivel de género, probablemente porque combina características de varios géneros reconocidos de los helechos cheilantoides. El indumento densamente viloso de las hojas, hasta ahora desconocido en Pellaea sect. Pellaea, está comúnmente asociado con taxa asignados a Cheilanthes (Tryon y Tryon, 1982). Sin embargo, otras características de Cheilanthes, tales como las hojas altamente divididas y los esporangios confinados a los engrosamientos de los márgenes de las venas modificadas (hidátodos marginales), están ausentes. Las hojas una vez pinnadas de $P$. ribae, extremadamente raras tanto en Pellaea como en Cheilanthes, se presentan en Astrolepis (Benham y Windham, 1992). Las escamas del rizoma, lineares, concoloras e irregularmente denticuladas, también son similares a las de Astrolepis, aunque las escamas se presentan en Pellaea atropurpurea (L.) Link, son comparables con la especie tipo de Pellaea. Otras probables sinapomorfias de Astrolepis (por ejemplo, escamas del indumento foliar peltadas, carencia de márgenes recurvados (falso indusio) de la pinna, 2 haces vasculares por pecíolo) excluyen a $P$. ribae de ser miembro en ese grupo. Es interesante notar, sin embargo, que el estudio molecular de secuencias rbcL e ITS (Gastony y Rollo, 1998) mostró que Astrolepis se encuentra dentro del grupo de Pellaea sensu-lato, que es parafilético. Así que no es inconcebible que un componente primitivo de Pellaea pueda exhibir algunas características típicamente presentes en Astrolepis. La información obtenida de las secuencias de ADN indican la necesidad de cambios mayores en los límites genéricos entre los helechos cheilantoides, hecho que puede afectar la disposición final de Pellaea ribae. La especie se asigna aquí a Pellaea con base en la preponderancia de los caracteres morfológicos.

Dentro de Pellaea, la nueva especie puede relacionarse con los miembros del grupo informal de "pecíolo-oscuro" de Tryon (1957). Es parecida a $P$. atropurpurea, el único representante con pubescencia de tipo viloso de la sección Pellaea previamente reconocido, el cual está ampliamente distribuido en México y los Estados Unidos (Tryon, 1968). También tiene pinnas no mucronadas y escamas del rizoma muy parecidas. Los dos taxa difieren en la densidad de la pubescencia, el grado de división de la hoja y la longitud del pedicelo 
esporangial. Pellaea ribae también tiene cierta semejanza con P. ternifolia subsp. villosa, un taxon principalmente mexicano, recientemente descrito por Windham (1993) a partir de una colecta de Hidalgo. Además de tener menos pubescencia y hojas pinnado-pinnatífidas, este taxon difiere de $P$. ribae por presentar escamas del rizoma bicoloras y pinnas mucronadas. Otras posibles relaciones de $P$. ribae en el grupo de pecíolo oscuro, incluyen a $P$. bridgesii Hook. (con pinnas enteras, no mucronadas y esporangios subsésiles) y $P$. breweri D.C. Eaton (con escamas del rizoma concoloras, esporangios subsésiles y pinnas no mucronadas). Es improbable un parentesco estrecho, dado que ambas especies son casi glabras y están confinadas a las altas montañas del oeste de los Estados Unidos.

Dada la dificultad de determinar relaciones entre los helechos cheilantoides únicamente sobre la base de datos morfológicos (ver Gastony y Rollo, 1998), sería prematuro discutir en este momento las posibles afinidades de Pellaea ribae. Esperamos que la continuación de los estudios de ADN de Pellaea y géneros relacionados ayude a establecer sus verdaderas relaciones filogenéticas. Lo que hasta el momento se sabe es que $P$. ribae es una especie muy singular y también muy rara, por lo que las instancias responsables de la protección de los recursos bióticos en México deberían enterarse de la existencia de esta planta única y hacer más trabajo de campo en San Luis Potosí y estados circunvecinos en un esfuerzo por localizar poblaciones adicionales para su conservación.

\section{AGRADECIMIENTOS}

Agradecemos a Adolfo Espejo y Ana Rosa López Ferrari la cuidadosa revisión del manuscrito, así como sus valiosas sugerencias y comentarios. A Felipe J. Madrigal Uribe y Gerald J. Gastony la traducción al latín de la diagnosis. A los curadores de los herbarios citados por el acceso a especímenes de Pellaea. El dibujo fue elaborado por Rolando Jiménez Machorro, la digitalización de la imagen por Jorge Lodigiani.

\section{LITERATURA CITADA}

Anthony, N. C. 1984. A revision of the southern African species of Cheilanthes Swartz and Pellaea Link (Pteridaceae). Contr. Bolus. Herb. 11: 1-291.

Benham, D. M. y M. D. Windham. 1992. Generic affinities of the star-scaled cloak ferns. Amer. Fern J. 82: 47-58.

Gastony, G. J. y D. R. Rollo. 1998. Cheilanthoid ferns (Pteridaceae: Cheilanthoideae) in the soutwestern United States and adjacent Mexico- a molecular phylogenetic reassessment of generic lines. Aliso 17: 131-144.

Tryon, A. F. 1957. A revision of the genus Pellaea section Pellaea. Ann. Missouri Bot. Gard. 44: 125193.

Tryon, A. F. 1968. Comparisons of sexual and apogamous races in the fern genus Pellaea. Rhodora 70: $1-24$.

Tryon, R. M. y A. F. Tryon. 1982. Ferns and allied plants, with special reference to tropical America. Springer-Verlag. Nueva York. i-xii, 857 pp.

Windham, M. D. 1993. New taxa and nomenclatural changes in the North American fern flora. Contr. Univ. Michigan Herb. 19: 31-61. 
Mendoza et al.: Una Nueva Especie de Pellaea del Estado de San Luis Potosí

Windham, M. D. 1993a. Pellaea Link. In: Flora of North America, North of Mexico. Vol. 2. Flora of North America Editorial Committee. Oxford University Press. Nueva York. pp. 175-186.

Recibido en julio de 2000. Aceptado en octubre de 2001. 